\title{
Pleomorphic lobular carcinoma of the breast: is it a prognostically significant pathological subtype independent of histological grade?
}

\author{
Emad A Rakha ${ }^{1,4}$, Carolien HM van Deurzen ${ }^{2,4}$, E Claire Paish ${ }^{1}$, R Douglas Macmillan ${ }^{3}$, \\ Ian O Ellis ${ }^{1}$ and Andrew HS Lee ${ }^{1}$ \\ ${ }^{1}$ Department of Histopathology, Nottingham University Hospitals, Nottingham, UK; ${ }^{2}$ Department of \\ Pathology, Erasmus Medical Center, Rotterdam, The Netherlands and ${ }^{3}$ Breast Institute, Nottingham \\ University Hospitals, Nottingham, UK
}

\begin{abstract}
Pleomorphic lobular carcinoma is regarded as a biologically aggressive variant of invasive lobular carcinoma of the breast. However, there is no consensus on the definition and whether this subtype adds useful information to histological grade. Two-hundred and two grade 2 or grade 3 invasive lobular carcinomas were studied. Tumours were categorised according to the components of histological grade: tubules, pleomorphism and mitoses. Pleomorphic lobular carcinoma was defined as a carcinoma with a lobular growth pattern and marked nuclear pleomorphism (pleomorphism 3). Breast cancer-specific survival was used in analysis of prognosis. Grade 3 pleomorphic lobular carcinomas (tubules 3, pleomorphism 3, mitoses 2 and tubules 3, pleomorphism 3, mitoses 3 ) had a worse prognosis than grade 2 (tubules 3, pleomorphism 2, mitoses 1) carcinomas. Grade 2 lobular carcinomas with marked nuclear pleomorphism (tubules 3, pleomorphism 3, mitoses 1) had a similar prognosis to grade 2 carcinomas with moderate pleomorphism (tubules 3, pleomorphism 2, mitoses 1 ). Survival was associated with mitotic score, but not with nuclear pleomorphism on both univariate and multivariate analysis. A non-classical growth pattern was seen more frequently in all subgroups with marked nuclear pleomorphism and was associated with worse survival. Histological grade and nodal status were independent of prognostic factors. This study shows that histological grade (in particular the mitotic component) in invasive lobular carcinomas is of prognostic importance, but pleomorphic type does not provide useful additional prognostic information.
\end{abstract}

Modern Pathology (2013) 26, 496-501; doi:10.1038/modpathol.2012.197; published online 14 December 2012

Keywords: breast; histological grade; pleomorphic lobular carcinoma; prognosis

Invasive lobular carcinoma is the commonest special type of breast cancer, representing about $10 \%$ of cases. It comprises several histological subtypes, all characterised by dyscohesive growth pattern and loss of function of the cell-adhesion protein E-cadherin. ${ }^{1-5}$ Pleomorphic lobular carcinoma was first described in 1987 by Page $^{6}$ as a variant of classical invasive lobular carcinoma. It is characterised by a classical growth pattern, but has marked nuclear atypia and frequently shows a plasmacytoid, histiocytoid or apocrine morphological appearance.

Correspondence: Dr AHS Lee, MD, MRCP, FRCPath, Department of Histopathology, Nottingham University Hospitals, City Hospital Campus, University of Nottingham, Hucknall Road, Nottingham NG5 1PB, UK.

E-mail: andrew.lee@nuh.nhs.uk

${ }^{4}$ These authors contributed equally to this paper.

Received 2 April 2012; revised 5 October 2012; accepted 5

October 2012; published online 14 December 2012
In contrast to the most common classical variant of invasive lobular carcinoma, which is typically strongly oestrogen receptor and progesterone receptor positive and ERBB2 (HER2) negative, the pleomorphic variant may express oestrogen receptor and progesterone receptor at lower levels and occasionally shows amplification of oncogenes, including ERBB2. ${ }^{7-9}$ Derksen et $a 1^{10}$ demonstrated that combined inactivation of E-cadherin and TP53 in mice induces development of invasive and metastatic mammary carcinomas, which resemble human pleomorphic lobular carcinoma. Previous studies, although limited by small numbers of patients, concluded that this subtype shows an aggressive clinical behaviour compared with classical invasive lobular carcinoma., $9,11,12$ One study reported a dismal outcome for pleomorphic lobular carcinoma with 9 out of 11 patients dying of their disease with a median survival of 2.1 years. ${ }^{12}$ These authors concluded based on this small 
number of cases that the outcome of pleomorphic lobular carcinoma is significantly worse than either infiltrating ductal carcinoma or classical invasive lobular carcinoma, even when stratified by axillary lymph node status. ${ }^{12}$ Pleomorphic lobular carcinomas have molecular genetic features characteristic of lobular carcinomas (loss of $16 q$ and gain of $1 \mathrm{q}$ ), but unlike the majority of classical invasive lobular carcinoma, they show additional genetic changes, such as ERBB2 amplification, more analogous to high-grade ductal carcinoma, which may be responsible for the more aggressive biological behaviour of pleomorphic lobular carcinoma. ${ }^{13,14}$

However, pleomorphic lobular carcinoma remains a poorly defined entity and there is no consensus on whether it is a distinct entity independent of histological grade. Several authorities, including the WHO Classification of Breast Tumours, described it as a variant of invasive lobular carcinoma that retains the distinctive growth pattern, but exhibits a greater degree of cellular atypia and pleomorphism than the classical form. 1,8,11 However, some pathologists and oncologists regard pleomorphic lobular carcinoma and high-grade (grade 3) invasive lobular carcinoma as synonymous, regardless of the nuclear features, mitotic counts, growth pattern or presence of apocrine or histiocytoid features. Historically, the value of histological grading of invasive lobular carcinoma according to Bloom and Richardson has been questioned because the majority of tumours are characterised by lack of tubule formation, moderate nuclear pleomorphism and limited mitotic activity, and are therefore grade 2. Although some studies reported limited prognostic value for histological grading of invasive lobular carcinoma, ${ }^{15}$ we and others have demonstrated that grading of these tumours, using the Nottingham grading system, is a strong and independent predictor of outcome, which supports the importance of routine assessment of histological grade in invasive lobular carcinoma. ${ }^{16-21}$ Analysing the three components of grading separately revealed that mitotic count was of more prognostic power than the two other components. ${ }^{16,21}$ This raises the question of whether pleomorphic lobular carcinoma as a distinct pathological subtype has any clinical value additional to grading, because conventional grading also includes cellular atypia and mitotic count as components. The aim of this study was therefore to evaluate the prognostic significance of pleomorphic lobular carcinoma and to provide recommendations regarding the relevance of using this separate subtype.

\section{Materials and methods}

\section{Patients}

A retrospective database of consecutive breast cancer patients was searched for patients with grade
2 or grade 3 invasive lobular carcinoma. These patients were diagnosed and treated at the Nottingham City Hospital from 1987-2010. Original slides of invasive lobular carcinoma classified as either mixed, solid, pleomorphic or grade 3 were retrieved and reviewed by specialized breast pathologists (CMHD, EAR and AHSL). Mixed lobular carcinoma was defined as a mixture of lobular subtypes with at least $10 \%$ of the second component. Carcinomas containing both lobular and ductal components were excluded; only pure lobular carcinomas were considered. Two-hundred and two grade 2 or grade 3 invasive lobular carcinomas were identified. Pleomorphic lobular carcinoma was defined as a carcinoma with a lobular growth pattern and marked nuclear pleomorphism (Figure 1). These carcinomas were compared with classical nonpleomorphic lobular carcinomas (tubules 3, pleomorphism 2, mitoses 1).

\section{Clinicopathological Features}

Clinicopathological features that were recorded included patient age, systemic treatment (endocrine and/or chemotherapy), tumour size, nodal status, grade (defined according to the modified BloomRichardson score based on tubule formation, nuclear pleomorphism and mitotic activity index), ${ }^{22}$ oestrogen receptor and ERBB2 status and prognosis. Oestrogen receptor positivity was defined as at least $10 \%$ of invasive carcinoma nuclei, showing immunohistochemical staining. ERBB2 staining was routinely performed in cases diagnosed after 2004. Cases diagnosed before 2000 were stained on tissue microarray sections using immunohistochemistry and chromogenic in situ hybridisation in borderline cases. E-cadherin was defined as positive if $1 \%$ or more invasive carcinoma cells showed membranous staining. Some of the tumours were stained as part of a previous study. ${ }^{2}$ Hormonal therapy was given to 107 patients and chemotherapy to 34 patients. None of the patients received adjuvant trastuzumab therapy.

Prognosis was expressed as breast cancer-specific survival and disease-free survival. Breast cancerspecific survival was defined as the interval from the operation to death from breast cancer. Diseasefree survival was calculated from the date of operation to the first recurrence (local, regional or distant).

\section{Statistical Analysis}

The $\chi^{2}$-test and the Student's $t$-test for trend were used to assess baseline differences between ordinal and continuous variables, respectively. The KaplanMeier method was used to estimate overall survival and disease-free survival. A Cox-proportional-hazards model was used to compare the cohorts and to adjust for known prognostic variables (tumour size, 

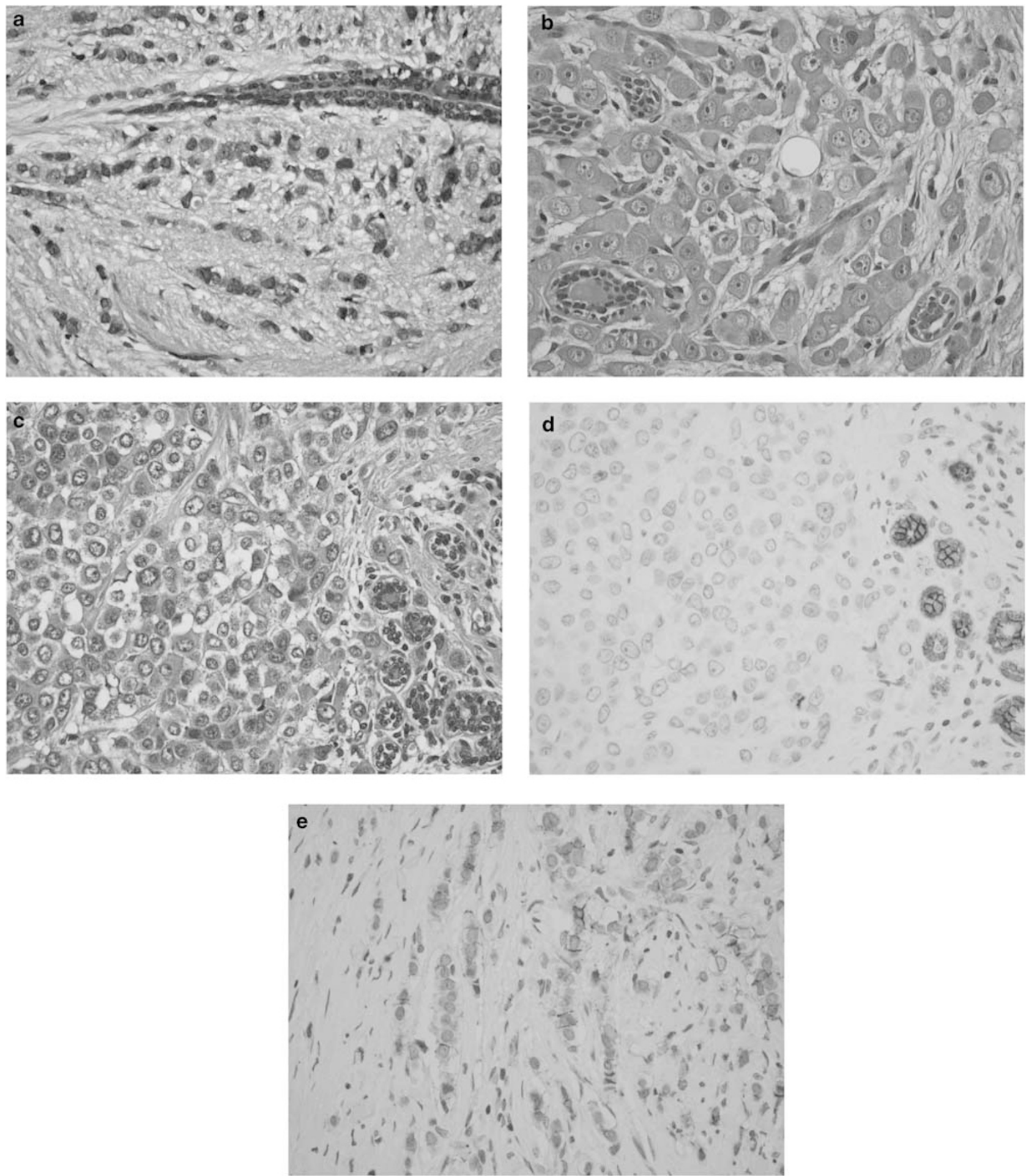

Figure 1 (a) Classical invasive lobular carcinoma with dyscohesive growth pattern and moderate nuclear pleomorphism. Pleomorphic lobular carcinomas (b) with classical growth pattern (c) with solid growth pattern (d) showing absence of E-cadherin expression and (e) showing patchy membranous E-cadherin. Note the membranous expression in normal breast epithelium in (d).

nodal status, vascular invasion, growth pattern and systemic therapy).

This study was approved by Nottingham Research Ethics Committee 2 under the title of 'Development of a molecular genetic classification of breast cancer'.

\section{Results}

The clinicopathological features of the invasive lobular carcinomas according to the subset of histological grade are summarised in Table 1. There were too few tubules 3 , pleomorphism 2 , mitoses 2 
$(n=7)$ and tubules 3, pleomorphism 2, mitoses 3 $(n=9)$ carcinomas for analysis. Compared with tubules 3, pleomorphism 2, mitoses 1 carcinomas, the non-classical growth patterns (mixed or solid) were more frequent in tubules 3 , pleomorphism 3 , mitoses $1\left(\chi^{2}=13, P=0.0003\right)$, tubules 3 , pleomorphism 3 , mitoses $2\left(\chi^{2}=72, P<0.0001\right)$ and tubules 3 , pleomorphism 3 , mitoses 3 carcinomas $\left(\chi^{2}=44, P<0.0001\right)$. The mitotic score component of grade was higher in carcinomas with solid or mixed than classical growth patterns $\left(\chi^{2}=65, P<0.0001\right.$ for both comparisons). Vascular invasion was more frequent in tubules 3 , pleomorphism 3 , mitoses 3 than tubules 3 , pleomorphism 2, mitoses 1 carcinomas $\left(\chi^{2}=8, P=0.004\right)$. Some pleomorphic lobular carcinomas had a central fibrotic focus, but we did not formally assess this feature. The majority (92\%) of cases were oestrogen receptor positive and $2 \%$ were positive for ERBB2, and the expression of these markers was not significantly different among the subsets. A total of $89 \%$ of carcinomas showed no E-cadherin expression with similar proportions in all subsets.

\section{Outcome}

The survival curves of patients with the four-grade subsets are shown in Figure 2. The median followup was 7.6 years (range 0.8-15.7) with 29 deaths because of breast cancer. Patients with tubules 3, pleomorphism 3, mitoses 1 carcinomas had a similar survival to patients with tubules 3 , pleomorphism 2 , mitoses 1 carcinomas $\left(\chi^{2}=0.7, P=0.39\right)$. Patients with tubules 3 , pleomorphism 3 , mitoses $3\left(\chi^{2}=4.9\right.$, $P=0.027$ ) or tubules 3 , pleomorphism 3 , mitoses 2 carcinomas $\left(\chi^{2}=11, P=0.0008\right)$ had a worse survival than those with tubules 3 , pleomorphism 2 , mitoses 1 carcinomas. There was no significant difference in survival between patients with tubules 3, pleomorphism 3, mitoses 3 and those with tubules 3 , pleomorphism 3 , mitoses 2 carcinomas $\left(\chi^{2}=0.26, P=0.61\right)$. If the analysis is restricted to patients with E-cadherin-negative carcinomas, the results are similar: patients with grade 3 carcinomas have a worse prognosis than those with tubules 3, pleomorphism 2, mitoses 1 carcinomas $\left(\chi^{2}=6.7, P=0.01\right)$ and the survival is similar for patients with tubules 3 , pleomorphism 3, mitoses 1 and tubules 3, pleomorphism 2, mitoses 1 carcinomas $\left(\chi^{2}=0.7, P=0.39\right)$.

On multivariate analysis of the pleomorphism and mitotic score components of histological grade as continuous variables, survival was associated with mitoses (relative risk 1.85 (95\% confidence intervals 1.16-2.95, $P=0.01$ ), but not with pleomorphism (relative risk 1.16 (95\% confidence intervals $0.52-$ 2.59, $P=0.72$ ). Carcinomas with a non-classical growth pattern had a worse survival compared with carcinomas with a classical growth pattern $\left(\chi^{2}=4.2\right.$, $P=0.04)$. On multivariate survival analysis,

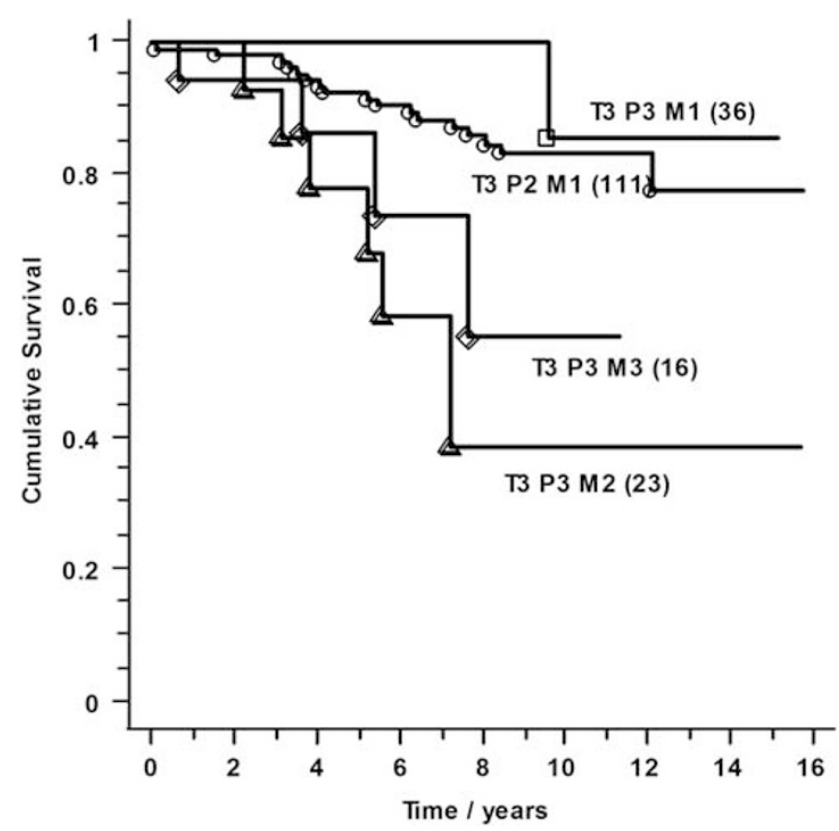

Figure 2 Breast cancer-specific survival in different grade subsets. The number of patients in each subset is indicated in brackets. ( $\mathrm{T}=$ tubules, $\mathrm{P}=$ pleomorphism and $\mathrm{M}=$ mitoses).

Table 1 Subsets of histological grade and relation to other pathological variables

\begin{tabular}{|c|c|c|c|c|c|c|c|c|c|}
\hline \multirow{2}{*}{$\begin{array}{l}\text { Grade } \\
\text { subset }\end{array}$} & \multirow{2}{*}{$\begin{array}{c}E R \\
\text { positive }\end{array}$} & \multirow{2}{*}{$\begin{array}{c}\text { ERBB2 } \\
\text { positive }\end{array}$} & \multicolumn{3}{|c|}{ Growth pattern } & \multirow{2}{*}{$\begin{array}{l}\text { Median size } \\
\quad(\mathrm{mm})\end{array}$} & \multirow{2}{*}{$\begin{array}{l}\text { Lymph node } \\
\text { positive }\end{array}$} & \multirow{2}{*}{$\begin{array}{c}\text { Vascular invasion } \\
\text { present }\end{array}$} & \multirow{2}{*}{$\begin{array}{l}\text { E-cadherin } \\
\text { negative }\end{array}$} \\
\hline & & & Classical & Mixed & Solid & & & & \\
\hline T3,P2,M1 & $\begin{array}{c}99 / 106 \\
(93 \%)\end{array}$ & $\begin{array}{l}1 / 111 \\
(1 \%)\end{array}$ & $\begin{array}{c}107 / 111 \\
(96 \%)\end{array}$ & $\begin{array}{c}4 / 111 \\
(4 \%)\end{array}$ & & 20 & $\begin{array}{c}45 / 110 \\
(41 \%)\end{array}$ & $\begin{array}{l}19 / 111 \\
(17 \%)\end{array}$ & $\begin{array}{c}99 / 111 \\
(89 \%)\end{array}$ \\
\hline T3,P3,M1 & $\begin{array}{l}26 / 30 \\
(87 \%)\end{array}$ & $\begin{array}{l}1 / 25 \\
(4 \%)\end{array}$ & $\begin{array}{l}27 / 36 \\
(75 \%)\end{array}$ & $\begin{array}{c}7 / 36 \\
(19 \%)\end{array}$ & $\begin{array}{l}2 / 36 \\
(6 \%)\end{array}$ & 22.5 & $\begin{array}{l}18 / 36 \\
(50 \%)\end{array}$ & $\begin{array}{c}6 / 34 \\
(18 \%)\end{array}$ & $\begin{array}{l}26 / 29 \\
(90 \%)\end{array}$ \\
\hline T3,P3,M2 & $\begin{array}{l}16 / 17 \\
(94 \%)\end{array}$ & $\begin{array}{l}1 / 14 \\
(7 \%)\end{array}$ & $\begin{array}{c}5 / 23 \\
(22 \%)\end{array}$ & $\begin{array}{l}12 / 23 \\
(52 \%)\end{array}$ & $\begin{array}{c}6 / 23 \\
(26 \%)\end{array}$ & 27 & $\begin{array}{c}9 / 22 \\
(41 \%)\end{array}$ & $\begin{array}{c}4 / 21 \\
(19 \%)\end{array}$ & $\begin{array}{l}18 / 20 \\
(90 \%)\end{array}$ \\
\hline T3,P3,M3 & $\begin{array}{l}12 / 14 \\
(86 \%)\end{array}$ & $0 / 9$ & $\begin{array}{c}6 / 16 \\
(37 \%)\end{array}$ & $\begin{array}{c}4 / 16 \\
(25 \%)\end{array}$ & $\begin{array}{c}6 / 16 \\
(37 \%)\end{array}$ & 26.5 & $\begin{array}{c}9 / 14 \\
(64 \%)\end{array}$ & $\begin{array}{c}8 / 15 \\
(53 \%)\end{array}$ & $\begin{array}{c}9 / 11 \\
(82 \%)\end{array}$ \\
\hline
\end{tabular}

Abbreviation: $\mathrm{ER}=$ oestrogen receptor. 
histological grade and nodal status were the only independent variables. Similar results were obtained for the above analyses with disease-free survival (data not shown).

\section{Discussion}

In the present study grade 3 invasive lobular carcinomas (tubules 3 , pleomorphism 3, mitoses 3 and tubules 3 , pleomorphism 3, mitoses 2) had a worse prognosis than grade 2 (tubules 3 , pleomorphism 2, mitoses 1) carcinomas. Grade 2 carcinomas with marked nuclear pleomorphism (tubules 3, pleomorphism 3, mitoses 1) had a similar survival to grade 2 carcinomas with moderate nuclear pleomorphism (tubules 3, pleomorphism 2, mitoses 1). On univariate and multivariate analysis, the mitotic count was associated with survival, but nuclear pleomorphism was not associated. This shows that mitoses are of more prognostic importance than pleomorphism and is consistent with studies of breast cancer as a whole. ${ }^{23}$ The number of patients in the present study is greater than previous studies of pleomorphic lobular carcinomas, but larger studies are needed to confirm or refute these observations and to examine the subsets with small numbers. Non-classical growth patterns were more frequent in carcinomas with marked nuclear pleomorphism. Talman et al ${ }^{17}$ reported that the majority of grade 3 invasive lobular carcinomas have a non-classical growth pattern and have a worse outcome than grade 2 carcinomas, irrespective of histological type. Histological grade was an independent prognostic factor in keeping with previous studies. ${ }^{18,21,24,25}$

In our series, we observed heterogeneity of pleomorphism in a substantial proportion of pleomorphic lobular carcinomas in both the in situ and the invasive components. This is consistent with recently published genetic data, suggesting that pleomorphic lobular carcinomas may evolve through a genetic pathway similar to that of classical invasive lobular carcinomas, but with the acquisition of additional genetic events. ${ }^{5,13,26}$

In the present study, about $90 \%$ of carcinomas in all the grade subsets were oestrogen receptor positive. Some studies have found that over 90\% of pleomorphic lobular carcinomas are oestrogen receptor positive, ${ }^{7,9}$ but others have shown a lower proportion of about $80 \% .^{13,27}$ The proportion of ERBB2-positive carcinomas was low in all subsets in the present study. Comparison with early studies is difficult as only immunohistochemistry was performed, but a recent study found ERBB2 amplification in $14 \%$ of pleomorphic lobular carcinomas. ${ }^{9}$ Our results are similar to previous studies of unselected invasive lobular carcinomas, which show that the majority of invasive lobular carcinomas (80-100\%) are oestrogen receptor positive and lack ERBB2 overexpression. ${ }^{13,18,21,28-30}$
We based the diagnosis of invasive lobular carcinoma on the typical dyscohesive growth pattern. We did not base it on E-cadherin expression as there is good evidence that about $10-15 \%$ of invasive lobular carcinomas show some membranous expression. ${ }^{2,31}$ Indeed it is possible for an invasive lobular carcinoma with a typical morphology to have an E-cadherin mutation, but show E-cadherin expression. ${ }^{31}$ Also, some nonlobular carcinomas lack E-cadherin expression. ${ }^{32}$ In the present study, about $10 \%$ of all the grade subsets showed some staining for E-cadherin. Most E-cadherin-positive lobular carcinomas show marked reduction or absence of expression of at least one of the catenins, ${ }^{2}$ consistent with the central role of loss of function of the the E-cadherin-catenin complex in this histological type. This absence of catenin expression is also seen in most E-cadherinpositive pleomorphic lobular carcinomas. If survival analysis is restricted to E-cadherin-negative carcinomas, the results are similar to the wholepatient group.

In conclusion, this study found that classification as the pleomorphic subtype added no useful additional prognostic information to histological grade in invasive lobular carcinomas. Histological grade should still be used for clinical decision making in invasive lobular carcinomas. Although the pleomorphic subtype is of biological interest, it does not appear to be of prognostic value.

\section{Disclosure/conflict of interest}

The authors declare no conflict of interest.

\section{References}

1 Ellis IO, Schnitt SJ, Sastre-Garau X, et al. Invasive breast carcinomas, In: Tavassoli FA, Devilee P(eds). Pathology and Genetics of Tumours of the Breast and Female Genital Organs. WHO Classification of Tumours. IARC press: Lyon, 2003, pp 13-59.

2 Rakha EA, Patel A, Powe DG, et al. Clinical and biological significance of E-cadherin protein expression in invasive lobular carcinoma of the breast. Am J Surg Pathol 2010;34:1472-1479.

3 Vos CB, Cleton-Jansen AM, Berx G, et al. E-cadherin inactivation in lobular carcinoma in situ of the breast: an early event in tumorigenesis. $\mathrm{Br} \mathrm{J}$ Cancer 1997;76:1131-1133.

4 Berx G, Cleton-Jansen AM, Nollet F, et al. E-cadherin is a tumour/invasion suppressor gene mutated in human lobular breast cancers. EMBO J 1995;14:6107-6115.

5 Weigelt B, Geyer FC, Natrajan R, et al. The molecular underpinning of lobular histological growth pattern: a genome-wide transcriptomic analysis of invasive lobular carcinomas and grade- and molecular subtypematched invasive ductal carcinomas of no special type. J Pathol 2010;220:45-57.

6 Page DL, Anderson TJ, Sakamoto G. Infiltrating carcinoma: major histological types, In: Page DL, 
Anderson TJ (eds). Diagnostic Histopathology of the Breast Edinburgh: Churchill Livingstone 1987, pp 193-235.

7 Frolik D, Caduff R, Varga Z. Pleomorphic lobular carcinoma of the breast: its cell kinetics, expression of oncogenes and tumour suppressor genes compared with invasive ductal carcinomas and classical infiltrating lobular carcinomas. Histopathology 2001;39: 503-513.

8 Radhi JM. Immunohistochemical analysis of pleomorphic lobular carcinoma: higher expression of p53 and chromogranin and lower expression of ER and PgR. Histopathology 2000;36:156-160.

9 Buchanan CL, Flynn LW, Murray MP, et al. Is pleomorphic lobular carcinoma really a distinct clinical entity? J Surg Oncol 2008;98:314-317.

10 Derksen PW, Braumuller TM, van der Burg E, et al. Mammary-specific inactivation of E-cadherin and p53 impairs functional gland development and leads to pleomorphic invasive lobular carcinoma in mice. Dis Model Mech 2011;4:347-358.

11 Weidner N, Semple JP. Pleomorphic variant of invasive lobular carcinoma of the breast. Hum Pathol 1992;23:1167-1171.

12 Bentz JS, Yassa N, Clayton F. Pleomorphic lobular carcinoma of the breast: clinicopathologic features of 12 cases. Mod Pathol 1998;11:814-822.

13 Simpson PT, Reis-Filho JS, Lambros MB, et al. Molecular profiling pleomorphic lobular carcinomas of the breast: evidence for a common molecular genetic pathway with classic lobular carcinomas. J Pathol 2008;215:231-244.

14 Reis-Filho JS, Simpson PT, Jones C, et al. Pleomorphic lobular carcinoma of the breast: role of comprehensive molecular pathology in characterization of an entity. J Pathol 2005;207:1-13.

15 Sinha PS, Bendall S, Bates T. Does routine grading of invasive lobular cancer of the breast have the same prognostic significance as for ductal cancers? Eur J Surg Oncol 2000;26:733-737.

16 Rakha EA, El-Sayed ME, Menon S, et al. Histologic grading is an independent prognostic factor in invasive lobular carcinoma of the breast. Breast Cancer Res Treat 2008;111:121-127.

17 Talman ML, Jensen MB, Rank F. Invasive lobular breast cancer. Prognostic significance of histological malignancy grading. Acta Oncol 2007;46:803-809.

18 Sastre-Garau X, Jouve M, Asselain B, et al. Infiltrating lobular carcinoma of the breast. Clinicopathologic analysis of 975 cases with reference to data on conservative therapy and metastatic patterns. Cancer 1996;77:113-120.
19 Pereira H, Pinder SE, Sibbering DM, et al. Pathological prognostic factors in breast cancer. IV: Should you be a typer or a grader? A comparative study of two histological prognostic features in operable breast carcinoma. Histopathology 1995;27:219-226.

20 Wachtel MS, Halldorsson A, Dissanaike S. Nottingham grades of lobular carcinoma lack the prognostic implications they bear for ductal carcinoma. J Surg Res 2010;166:19-27.

21 Bane AL, Tjan S, Parkes RK, et al. Invasive lobular carcinoma: to grade or not to grade. Mod Pathol 2005;18:621-628.

22 Elston CW, Ellis IO. Pathological prognostic factors in breast cancer I. The value of histological grade in breast cancer: experience from a large study with longterm follow-up. Histopathology 1991;19:403-410.

23 Rakha EA, El-Sayed ME, Lee AHS, et al. Prognostic significance of Nottingham histologic grade in invasive breast carcinoma. J Clin Oncol 2008;26:3153-3158.

24 Moreno-Elola A, Aguilar A, Roman JM, et al. Prognostic factors in invasive lobular carcinoma of the breast: a multivariate analysis. A multicentre study after seventeen years of follow-up. Ann Chir Gynaecol 1999;88:252-258.

25 Mokbel K. Grading of infiltrating lobular carcinoma. Eur J Surg Oncol 2001;27:609-610.

26 Chen YY, Hwang ES, Roy R, et al. Genetic and phenotypic characteristics of pleomorphic lobular carcinoma in situ of the breast. Am J Surg Pathol 2008;33:1683-1694.

27 Middleton LP, Palacios DM, Bryant BR, et al. Pleomorphic lobular carcinoma: morphology, immunohistochemistry, and molecular analysis. Am J Surg Pathol 2000;24:1650-1656.

28 Rakha EA, El-Sayed ME, Powe DG, et al. Invasive lobular carcinoma of the breast: response to hormonal therapy and outcomes. Eur J Cancer 2008;44:73-83.

29 Arpino G, Bardou VJ, Clark GM, et al. Infiltrating lobular carcinoma of the breast: tumor characteristics and clinical outcome. Breast Cancer Res 2004;6:R149-R156.

30 Gonzalez-Angulo AM, Sahin A, Krishnamurthy S, et al. Biologic markers in axillary node-negative breast cancer: differential expression in invasive ductal carcinoma versus invasive lobular carcinoma. Clin Breast Cancer 2006;7:396-400.

31 Da Silva L, Parry S, Reid L, et al. Aberrant expression of E-cadherin in lobular carcinomas of the breast. Am J Surg Pathol 2008;32:773-783.

32 Rakha EA, Abd El Rehim D, Pinder SE, et al. E-cadherin expression in invasive non-lobular carcinoma of the breast and its prognostic significance. Histopathology 2005;46:685-693. 